\title{
O QUE INFLUENCIA A PERFORMANCE ESTUDANTIL NO ENADE? INVESTIGAÇÃO QUANTITATIVA DOS CURSOS PÚBLICOS DE ENGENHARIA CIVIL NO BRASIL
}

\author{
Gabriel Villas Boas de Amorim Lima-gabrielvbal@gmail.com* \\ Programa de Pós-Graduação em Engenharia Civil, Universidade Federal do Pará* \\ Rua Augusto Correa, 01 * \\ 66075-110-Belém-Pará*
}

Ana Catarina Gandra de Carvalho - anacatarinagandra@gmail.com

Renato Martins das Neves - rmdasneves@gmail.com

\begin{abstract}
Resumo: Este estudo objetivou identificar quais fatores institucionais influenciam no desempenho de concluintes de engenharia civil, oriundos de instituições públicas, no seu desempenho no ENADE. Métodos inferenciais envolvendo regressão linear múltipla foram adotados para modelar a interação de 9 variáveis associadas à docência e à instituição concedente em 119 programas do todo o Brasil. Após a validação estatística do modelo, 5 variáveis associadas à qualificação docente, didática docente, tempo de funcionamento do curso, organização acadêmica e jurisdição administrativa mostraram-se correlatas à variação no desempenho estudantil no ENADE. Variáveis não validadas pelo modelo como dedicação docente, número de vagas, carga-horária curricular e regime de oferta não influenciam diretamente no desempenho estudantil no ENADE, apesar da potencial interferência no desempenho dos estudantes durante a graduação. As contribuições teóricas desta pesquisa para o campo de abordagens quantitativas do desempenho de estudantes de engenharia no ENADE são inegáveis, além de contribuir em termos práticos para o desenvolvimento de políticas educacionais de avaliação de cursos de educação superior em engenharia pelo Brasil.
\end{abstract}

Palavras-chave: Educação em engenharia civil. Regressão linear. ENADE.

\section{INTRODUÇÃO}

A engenharia civil desempenha papel fundamental na sociedade, demandando de mão-deobra qualificada para atender às exigências técnicas da profissão. Contudo, a formação deficitária dos engenheiros civis é uma realidade enfrentada pelo mercado brasileiro, cujos profissionais formados tendem a carecer de conhecimentos, habilidades e competências que deveriam ter sido desenvolvidas à época da formação universitária (CORDEIRO et al., 2008).

Tais deficiências são muitas vezes evidentes no desempenho dos estudantes no ENADE (Exame Nacional de Desempenho dos Estudantes), principal instrumento utilizado pelo Ministério da Educação (MEC) para avaliar os cursos de graduação do país. Aplicado trienalmente para cada área do conhecimento, o ENADE avalia tanto conhecimentos gerais quanto específicos dos concluintes, cuja nota ponderada é agrupada em 5 faixas de classificação (MOREIRA, 2010). 
Ao longo de cada edição, um conceito é gerado para cada programa e aqueles que obtém consecutivamente nota abaixo de 3 são indicados para desligamento da autorização governamental (SILVA, 2017). Contudo, não somente aspectos psicológicos, sociais e culturais dos estudantes estão associados ao seu desempenho no exame, como também fatores institucionais, ligados ao papel do professor e da universidade. Mas como tais fatores afetam o desempenho dos concluintes no ENADE?

Pensando nisso, este estudo objetivou identificar quais fatores institucionais influenciam no desempenho de concluintes de engenharia civil, oriundos de instituições públicas, no seu desempenho no ENADE. A restrição à esfera pública de ensino agrega valor ao estudo ao direcionar a pesquisa para instituições que de fato possam ter intervenções por parte dos órgãos competentes, como Secretarias Municipais, Estaduais e Ministério da Educação (MEC). Também se limitou a investigar somente os fatores institucionais com o intuito de direcionar a pesquisa na compreensão mais aprofundada da natureza desses preditores.

Para alcançar estes objetivos, métodos estatísticos inferenciais foram aplicados, validando seus modelos através de protocolos técnicos consolidados. Os dados foram extraídos de plataformas oficiais do governo e da imprensa nacional, o qual resultaram em um banco de dados próprio deste estudo. Os resultados foram discutidos do ponto de visto analítico e as discussões foram embasadas em prospecções já evidenciadas na literatura.

Esta pesquisa contribui para a literatura de abordagens inferenciais na investigação pedagógica de desempenho estudantil por parte de cursos de engenharia, em especial a civil. A abordagem se diferencia da literatura atual quanto à robusta validação dos resultados e à discussão transversal das predições. Também se contribui para a prática de planejadores educacionais, no auxílio à formação de protocolos de avaliação de cursos de graduação no Brasil, especialmente nos de engenharia, salientando os aspectos mais importantes e críveis a serem considerados, evidenciando uma análise mais racional do desempenho acadêmico.

\section{MÉTODO}

\subsection{Descrição e Coleta dos dados}

As variáveis utilizadas neste estudo estão descritas no Quadro 1. A variável ENADE representa o valor contínuo do ENADE obtido por cada curso e será modelada como a variável dependente (a ser explicada pelo modelo inferencial). Apesar do ENADE ser discutido por faixas em relatórios oficiais, seu valor contínuo é o mais adequado para tratamentos estatísticos, por possibilitar uma maior variabilidade na amostra, representando a performance mais fidedigna dos programas. Essa variável foi extraída do portal oficial do Instituto Nacional de Estudos e Pesquisas Educacionais Anísio Teixeira (INEP, 2017), referente à edição ENADE 2017, cujo relatório é o mais atualizado disponível. Apenas cursos que realizaram o ENADE 2017 foram considerados no banco de dados resultante.

\begin{tabular}{|c|c|l|c|c|}
\hline Variável & $\begin{array}{c}\text { Tipo de } \\
\text { variável }\end{array}$ & \multicolumn{1}{|c|}{ Descrição } & intervalo & unidade \\
\hline ENADE & contínua & $\begin{array}{l}\text { Pontuação geral do curso obtida } \\
\text { pelos concluintes no ENADE 2017 }\end{array}$ & $0-5,00$ & pontos \\
\hline Didática & contínua & $\begin{array}{l}\text { Percepção do RUF 2017 sobre a } \\
\text { didática dos professores }\end{array}$ & $0-100,00$ & pontos \\
\hline Qualificação & contínua & $\begin{array}{l}\text { Percepção do RUF 2017 sobre a } \\
\text { qualificação dos professores }\end{array}$ & $0-8,00$ & pontos \\
\hline
\end{tabular}


"Os desafios para formar hoje o engenheiro do amanhã"

\begin{tabular}{|c|c|c|c|c|}
\hline Dedicação & contínua & $\begin{array}{l}\text { Percepção do RUF } 2017 \text { sobre a } \\
\text { dedicação dos professores }\end{array}$ & $0-8,00$ & pontos \\
\hline Vagas & contínua & $\begin{array}{l}\text { Número de vagas disponibilizadas } \\
\text { em cada processo seletivo }\end{array}$ & - & alunos \\
\hline Currículo & contínua & Carga horária total do curso & - & horas-aula \\
\hline Tempo & contínua & Tempo de funcionamento do curso & - & anos \\
\hline Oferta & dicotômica & $\begin{array}{l}\text { Regime de oferta das disciplinas do } \\
\text { curso }\end{array}$ & - & $\begin{array}{l}\text { Não anual (1) } \\
\text { anual (0) }\end{array}$ \\
\hline Organização & dicotômica & $\begin{array}{l}\text { Porte da instituição concedente do } \\
\text { curso }\end{array}$ & - & $\begin{array}{c}\text { universidade } \\
\text { (1); não é } \\
\text { universidade } \\
(0)\end{array}$ \\
\hline Jurisdição & categórica & $\begin{array}{l}\text { Nível governamental de } \\
\text { administração da instituição } \\
\text { concedente }\end{array}$ & - & $\begin{array}{c}\text { Municipal (1), } \\
\text { estadual (2), } \\
\text { federal (3) }\end{array}$ \\
\hline
\end{tabular}

Fonte: Autores (2020), com base nos arquivos digitais do INEP (2017), RUF (2017) e e-MEC (2020)

As variáveis independentes (preditores, ou variáveis que irão tentar explicar o comportamento da variável dependente) foram agrupadas em 2 grupos: I) atributos dos docentes e; II) atributos da instituição. As variáveis do primeiro grupo são: Didática docente (Didática); Qualificação docente (Qualificação); e Dedicação docente (Dedicação). Essas variáveis foram obtidas do Ranking Universitário da Folha (RUF, 2017), um relatório anual elaborado pelo Jornal Folha de São Paulo, que avalia o desempenho dos cursos de graduação no Brasil. As variáveis do segundo grupo formam os aspectos associados à instituição ofertante: Número de vagas ofertadas (Vagas), Carga-horária curricular total (Currículo), Tempo de funcionamento de curso (Tempo); Regime de oferta das disciplinas (Oferta), Organização acadêmica (Organização) e Jurisdição administrativa (Jurisdição). Essas 7 variáveis foram extraídas do portal oficial do MEC (e-MEC), que disponibiliza informações de todos os cursos de graduação cadastrados no país.

\subsection{Análise dos dados}

A técnica inferencial adotada neste estudo foi a regressão linear múltipla (RLM). Em modelos de regressão, propõe-se estimar o comportamento de uma variável dependente (neste caso, $E N A D E$ ) a partir da interação desta com uma (regressão simples) ou várias variáveis simultaneamente (regressão múltipla), sendo estas denominadas variáveis independentes, ou preditoras. As predições podem adotar conformações lineares ou não lineares, a depender da natureza da variável dependente e das características da amostra (HAIR et al., 2018).

Dentre os diversos modelos de RLM existentes, a técnica dos Mínimos Quadrados Ordinários (MQO) foi considerada a mais compatível com a natureza contínua da variável dependente. Este modelo prediz que as variáveis independentes não devem ter poder explicativo entre si, isto é, não devem se sobrepor na explicação da variável dependente, sendo este fenômeno denominado multicolinearidade, cuja ocorrência induz a estimativas incorretas nas predições. A ocorrência de multicolinearidade é detectada através do teste VIF (variance inflactor fator), que descarta sua ocorrência caso o VIF seja menor que 5.00 (CLEFF, 2019).

O modelo MQO também prediz que os resíduos da regressão (isto é, a parcela estocástica do modelo) devam ser normais e homocedásticos. A normalidade dos resíduos indica que as predições de significância das variáveis podem ser corroboradas, ao passo que a 
homocedasticidade indica que os resíduos mantêm uma variância constante, duas das premissas para se validar um modelo MQO (HAIR et al., 2018). A normalidade foi verificada com o teste de Shapiro-Wilk W., que indica o valor mínimo de 0,0500 para a aceitabilidade do modelo. Já a homocedasticidade foi verificada através do teste Breusch-Pagan, que valida regressões cuja significância estatística do $\chi^{2}$ seja maior que 0,0500 (CLEFF, 2019).

Uma vez confirmada a inexistência de multicolinearidade, não-normalidade e heterocedasticidade, os resultados da regressão podem ser analisados. Parâmetros da regressão e ajustamento geral do modelo adotaram significância estatística de 5\%. Este modelo inferencial possui dois objetivos: I) verificar se fatores institucionais conseguem explicar o desempenho estudantil no ENADE e; II) identificar fatores institucionais estatisticamente significantes, hierarquizando suas influências por meio dos coeficientes Beta.

A Hipótese zero $\left(\mathrm{H}_{0}\right)$ considera que os fatores institucionais descritos neste estudo não influenciam o desempenho dos estudantes, enquanto que a Hipótese $1\left(\mathrm{H}_{1}\right)$ corrobora sua influencia estatística. Em caso de $\mathrm{H}_{1}$ ser confirmada, os fatores que puderem ser validados pela regressão serão hierarquizados por meio de seus coeficientes Beta, que são os coeficientes ordinários de regressão padronizados pelos seus respectivos testes T. A padronização dos coeficientes permite a comparação entre variáveis com escalas diferentes, possibilitando uma hierarquização de influências estatísticas (HAIR et al., 2018). Todos os testes foram conduzidos no software Stata versão 13.0.

\section{RESULTADOS}

\subsection{Diagnóstico descritivo}

O banco de dados resultante é constituído de 119 cursos de engenharia civil de instituições públicas brasileiras. A amostra indica que os fatores docentes e a carga-horária curricular apresentaram as menores variabilidades, enquanto que os demais fatores ligados à instituição de ensino se apresentaram dispersos. A didática docente apresentou uma variação entre 8,15 e 61,74 pontos, média de 24,84 e desvio padrão de 12,58. Valores de desvio padrão menores que os médios indicam uma concentração analítica da amostra abaixo da média nacional, sugerindo uma deficiência na didática de ensino. Já os fatores de qualificação e dedicação docente mostram valores mais expressivos, com variações entre 0,80 e 8,00; e 0,18 e 8,00 , respectivamente. Ambos os fatores estão concentrados, todavia, acima dos valores médios, com desvios-padrão de 1,88 e 1,45; e médias de 6,10 e 7,39, respectivamente. As crescentes tendências de obrigatoriedade de titulação e dedicação exclusiva (especialmente em instituições federais) podem explicar tal comportamento positivo.

O número de vagas ofertadas variou de 17 a 310 alunos admitidos por processo seletivo, com média nacional de 79,30 e desvio-padrão de 46,33 alunos. Isso indica que a maioria dos cursos ofertam entre 17 e 79 vagas por processo seletivo. A carga-horária curricular demonstrou uma variabilidade entre 3.306 e 5.120 horas, com media de 4.116 horas e desviopadrão de 371,74 alunos. A concentração deste indicador entre 3.306 e 4.116 horas pode ser explicada pela obrigatoriedade de carga-horária mínima exigida pelo MEC para cursos de engenharia civil ser de 3.300 horas; enquanto que cursos com duração superior a 4.100 horas podem implicar em maiores custos de mão-de-obra (técnica e docente), recursos e infraestrutura.

O regime de oferta indicou que $89,08 \%$ das disciplinas são ofertadas de forma semestral ou trimestral, enquanto que apenas $10,92 \%$ são ofertadas na modalidade anual. $O$ regime fracionado é preferido por instituições federais que buscam maior flexibilização do currículo, com maior possibilidade de escolha do aluno em áreas de ênfase. Também são considerados fatores como mobilidade discente e maior dinâmica de atividades de pesquisa. Já cursos anuais são desenvolvidos em programas que almejam uma formação mais próxima entre 
professor e aluno, já que o tempo de desenvolvimento das atividades curriculares é dilatado. Este método é mais aplicado em cursos que disponibilizam oficinais interdisciplinares.

A organização acadêmica indicou que $78,99 \%$ dos cursos públicos de engenharia civil são ofertados pelas universidades, enquanto que $21,01 \%$ são ofertados por institutos, centros universitários e faculdades. A jurisdição administrativa complementa que instituições federais representam $69,75 \%$ de todos os cursos públicos do Brasil, seguido pelas instituições estaduais $(24,37 \%)$ e municipais $(5,88 \%)$. É possível depreender deste diagnóstico que os cursos públicos de engenharia civil estão concentrados em universidades federais, que ainda representam fortemente a educação de engenharia civil no Brasil.

\subsection{Diagnóstico inferencial}

Os resultados da regressão estão discriminados na Tabela 1. O teste $\mathrm{F}$ (Prob $>\mathrm{F}=$ 0,0000) indicou que a Hipótese $\mathrm{H}_{0}$ pode ser rejeitada com alta confiabilidade estatística $(99,9999 \%)$, atestando que os fatores institucionais afetam o desempenho estudantil no ENADE. Logo, a Hipótese $\mathrm{H}_{1}$ é confirmada. Ademais, as ausências de multicolinearidade (VIF médio $=1,67$ ), não-normalidade (Prob $>\mathrm{F}=0,5369$ ) e heterocedasticidade (Prob $>\chi^{2}=$ 0,2741 ) foram confirmadas, indicando que o modelo MQO possui confiabilidade estatística e que a amostra possui robustez inferencial.

Tabela 1 - Regressão linear múltipla

\begin{tabular}{c|c|c|c|c|}
\hline \multicolumn{6}{l}{ Variável dependente: ENADE } & \multicolumn{1}{|l|}{$\mathbf{p} \mathbf{t} \mid$} & Beta \\
\hline Variável & Coeficiente & $\mathbf{t}$ & 0,010 & 0,2251 \\
\hline Didática & 0,0138 & 2,61 & 0,000 & 0,3939 \\
\hline Qualificação & 0,1620 & 3,98 & 0,878 & 0,1236 \\
\hline Dedicação & 0,0066 & 0,15 & 0,145 & $-0,1403$ \\
\hline Vagas & $-0,0023$ & $-1,47$ & 0,743 & $-0,0235$ \\
\hline Currículo & $-0,0000$ & $-0,33$ & 0,039 & 0,1812 \\
\hline Tempo & 0,0043 & 2,09 & 0,713 & 0,0278 \\
\hline Oferta & 0,0686 & 0,37 & 0,008 & $-0,2322$ \\
\hline Organização & $-0,4381$ & $-2,72$ & & \\
\hline Jurisdição & & & 0,002 & 0,5166 \\
\hline Estadual & 0,9251 & 3,22 & 0,002 & 0,5201 \\
\hline Federal & 0,8705 & 3,16 & 0,037 & - \\
\hline Constante & 1,6804 & 2,11 &
\end{tabular}

Fonte: Autores (2020)

Uma vez validada a regressão, seus coeficientes e ajustamentos puderam ser discriminados. Dos 9 preditores modelados, 5 foram validados estatisticamente (Didática, Qualificação, Tempo, Organização e Jurisdição), além da constante de regressão, cuja significância também é considerada uma premissa de aceitabilidade do modelo MQO. O coeficiente do preditor Didática indica que para cada 1,00 ponto acrescido na didática docente, é esperado um aumento de 0,0138 pontos no desempenho do ENADE, enquanto que o preditor Qualificação indica que para cada 1,00 ponto acrescido na qualificação docente, é previsto um aumento de 0,1620 pontos na nota do ENADE. O preditor Dedicação não obteve significância estatística, indicando que a dedicação docente não é válida para estimar a nota dos estudantes no ENADE.

O preditor Tempo evidenciou uma correlação positiva, indicando que para cada 1 ano de operação do curso de engenharia civil, é esperado que a sua nota no ENADE aumente 0,0043 pontos. Já para o preditor Organização, a relação é inversa. Considerando que esta variável é 
dicotômica, infere-se da regressão que cursos ofertados por universidades tendem a ter um desempenho inferior àqueles ofertados por institutos, centros universitários e faculdades em 0,4381 pontos. O preditor Jurisdição, como variável categórica, é analisado entre suas categorias internas a partir um fator de referência gerado pelo próprio modelo. Tendo como referência instituições municipais (1), a regressão induz que cursos de instituições federais e estaduais tendem a ter 0,9251 e 0,8705 pontos a mais no ENADE do que cursos de instituições municipais, respectivamente. Os preditores Vagas, Currículo e Oferta não demonstraram significância estatística, logo, não influenciam na nota do ENADE.

Ao analisar os preditores válidos por meio de seus coeficientes Beta, indicou-se que Jurisdição é o fator que mais influencia na nota do ENADE, especialmente se o curso for ofertado por uma instituição federal $(0,5201)$ ou estadual $(0,5166)$. Em seguida, fatores como Qualificação $(0,3939)$, Organização $(-0,2322)$, Didática $(0,2251)$ e Tempo $(0,1812)$ puderam ser hierarquizados. O ajustamento do modelo indicou poder de explicação aceitável $\left(\mathrm{R}^{2}\right.$ ajustado $=0,4850$ ) para estudos em ciências sociais aplicadas (CLEFF, 2019). Com isso, percebe-se que tais fatores institucionais são responsáveis por explicar 48,50\% da variação do desempenho de alunos de engenharia civil no ENADE 2017. Outros fatores, de natureza socioeconômica, demográfica, psicológica e geográfica podem estar associados à parcela cuja modelo não consegue predizer, contudo, tais investigações fogem ao escopo deste estudo.

\section{DISCUSSÕES}

\subsection{Fatores institucionais ligados à docência}

Dos 3 fatores institucionais ligados à prática docente, Qualificação e Didática mostraram correlação estatisticamente significante com o desempenho estudantil no ENADE. Tais correlações também foram encontradas por Moreira (2010) para o curso de engenharia civil à época. Este resultado implica que tanto a especialização técnica quanto o emprego adequado de recursos pedagógicos são essenciais na garantia de um desempenho satisfatório dos estudantes. Isso evidencia a necessidade de o corpo docente aliar conhecimento e habilidades demandados pela engenharia com métodos e abordagens didáticas que forneçam as condições necessárias para o cumprimento dos objetivos de ensino-aprendizagem. Nesse ponto de vista, a figura do engenheiro educador nunca esteve tão presente e necessária no contexto do ensino da engenharia civil, de modo que somente o conhecimento técnico não é mais suficiente para garantir um ensino de qualidade, reflexo este no desempenho estudantil (CORDEIRO, 2008).

A especialização do docente, que remota dos anos 1980, surgiu a partir da necessidade de conciliar conhecimentos práticos com metodologias e abordagens inerentes à prática científica, vivenciada através de cursos de pós-graduação de nível strictu sensu. Antes dessa macrotendência universitária, a figura do professor era associada à prática profissional consolidada, de modo que a excelência no mercado de trabalho era comumente associada a boas práticas de ensino. Dessa forma, a experiência profissional docente era sobreposta à sistematização do método científico, relativizando o conhecimento técnico ao empirismo que provocavam fragilidades sistêmicas na construção do conhecimento (LEITÃO, 2001).

Além disso, práticas didáticas eram rudimentares e pouco se discutia habilidades e competências do engenheiro egresso. Com a gradual qualificação do corpo docente (especialmente em nível federal), práticas científicas tornaram-se balizadoras na estruturação pedagógica de cursos de engenharia civil, sistematizando e universalizando conhecimentos e técnicas da área. Todavia, aspetos relacionados à didática e ao desenvolvimento de habilidades e competências ainda não satisfazem critérios aceitáveis na realidade brasileira.

Isso é evidenciado pelo baixo desempenho didático encontrado na amostra analisada, revelando a necessidade de os docentes de engenharia civil desenvolverem mais habilidades e competências didáticas, seja por incorporação de conteúdos pedagógicos no escopo de pós- 
graduações em engenharia civil (já que as mesmas formam os futuros professores) ou por capacitações específicas. A exigência de comprovação de capacitação pedagógica como prérequisito para ingresso no magistério superior e a comprovação periódica de atualizações capacitadoras em didática mostram-se soluções críveis para mitigar o baixo desenvolvimento didático dos professores.

O tempo de dedicação docente não apresentou influência direta no desempenho estudantil no ENADE, o que não descarta sua possível interferência no desempenho dos alunos dentro da própria graduação. Professores com dedicação exclusiva estão mais suscetíveis a participar de projetos de ensino, pesquisa e extensão - participando de forma mais ativa no desenvolvimento dos cursos e dos alunos (SANKARAN; MOHANTY, 2018). A participação mais presente dos professores indica também maior foco à produção acadêmica, científica e maior disponibilidade para desenvolver habilidades e competências pedagógicas.

\subsection{Fatores institucionais ligados à instituição}

Dos 6 fatores institucionais ligados à instituição, Tempo, Organização e Jurisdição mostraram correlação estatisticamente significativa com o desempenho estudantil no ENADE. Tais correlações também foram encontradas por Moreira (2010) para o curso de engenharia civil à época. Este resultado implica que o porte e a institucionalização dos cursos de engenharia civil afetam na performance estudantil, assim como a tradição de ensino. A regressão induz que cursos mais antigos tendem a prover melhores resultados no ENADE. Na amostra, 6 cursos que estão em funcionamento há pelo menos 100 anos coincidentemente obtiveram excelentes performances no ENADE 2017. Essa correlação pode estar associada ao fato de que escolas mais antigas possuem mais tempo para introduzir e adaptar novos métodos e abordagens de ensino, otimizando sua operabilidade à demanda estudantil e ao escopo técnico exigido, atingindo níveis de excelência.

A organização acadêmica também revela ser um fator influenciador do desempenho no ENADE, de modo que instituições de menor porte (como institutos, centros universitários e faculdades) tendem a ter melhores desempenhos do que grandes instituições (universidades). Isso ocorre, pois, a variabilidade de desempenho entre as universidades é muito maior do que entre instituições menores, isto é, existem cursos de universidades com excelentes performances no ENADE e cursos com performances insatisfatórias. Isso demonstra que o desempenho é fragmentado entre instituições de mesmo nível organizacional, revelando disparidades institucionais evidentes.

Exemplos são vistos quando as melhores universidades federais se concentram nas regiões Sul e Sudeste do Brasil enquanto que os piores desempenhos são observados na região Norte. Entre as instituições menores, a variabilidade é supreendentemente menor, demonstrando maior uniformidade no desempenho estudantil. Isso não quer dizer que todas as instituições menores sejam melhores que as universidades, mas sim que a tendência de discrepância entre elas é menor, suscetibilizando-as a ter predições mais precisas no ENADE.

Com relação à jurisdição administrativa, a regressão indicou que instituições federais e estaduais possuem maior desempenho que instituições municipais, o que a princípio se mostra lógico. Instituições federais geralmente recebem mais recursos da União, que são transformados em melhores condições de infraestrutura, incentivo à pesquisa e extensão e à melhor contratação de mão-de-obra qualificada. Instituições estaduais, e principalmente municiais, recebem recursos em escalas proporcionais às receitas de suas esferas, refletindo diretamente no auxílio necessário ao desenvolvimento das práticas educacionais. Salvo pontuais realidades de estados e municípios brasileiros, a maioria da amostra reflete a carência de recursos necessários para o aprimoramento do ensino superior, em especial o de engenharia civil, que demanda de aparato físico e tecnológico de apoio. 
Apesar de não serem estatisticamente significantes para explicar o desempenho estudantil no ENADE, Vagas, Currículo e Oferta podem influenciar indiretamente o desempenho estudantil ao longo da graduação. O número de vagas autorizadas é proporcional à capacidade gerencial e orçamentária do curso e da instituição concedente, dependendo de grandes recursos infraestruturais, laborais e financeiros a depender do número de calouros por período letivo. Com isso, o número de vagas ingressantes é diretamente proporcional às condições de operabilidade da graduação, sendo esta um importante medidor de qualidade. Para ter bons desempenhos, um curso deve prover recursos mínimos de operação, muitas vezes negligenciados em função de decisões gerenciais desalinhadas com as práticas pedagógicas.

Já o desenho curricular, expresso quantitativamente em horas-aula, influencia na abrangência e abordagem sistemática e sistêmica que o curso opera (RIVERA-RODRIGUEZ, 2019). Apesar de promoverem uma única formação profissional, a mesma graduação com carga-horárias discrepantes oferece perspectivas e entendimentos distintos da natureza do ofício aprendido, refletindo futuramente no exercício profissional. Seja pela maior quantidade de conteúdos abordados ou pelo aprofundamento de percepção dos eixos temáticos, maiores cargas-horárias podem induzir uma melhor compreensão dos conhecimentos, recursos e competências necessárias da profissão.

Por outro lado, o excesso de tempo em sala de aula pode e deve ser questionado, já que o mesmo não se provou eficiente na determinação de sucesso de egressos em um exame técnico. Práticas pedagógicas mais recentes evidenciam o aprendizado focado no aluno, de modo que o conteúdo seja introduzido em aula e o aprofundamento seja realizado pelo próprio estudante, que detém a iniciativa em buscar conhecimento. Metodologias baseadas na resolução de problemas (FORCAEL et al., 2015) mostram-se soluções eficientes à altas cargas-horárias atuais para obter bons desempenhos acadêmicos. Além disso, o ensino integral também pode implicar na exclusão de alunos hipossuficientes, que demandam de parte da carga horária diária para desenvolver atividades profissionais e prover familiarmente (PEREIRA, 2017).

Quanto ao regime de oferta, suas implicações também estão associadas ao desenvolvimento de atividades dentro da própria graduação. Cursos trimestrais e semestrais buscam maior flexibilidade, disponibilidade e formas de abordagens do conteúdo, além de possibilitar ao aluno melhor customização da formação profissional por meio de suas afinidades acadêmicas. Já cursos anuais implicam em maior ênfase no processo de ensinoaprendizagem, de modo que a relação professor e aluno torna-se mais sólida ao desenvolver projetos, experimentos e atividades durante um cronograma mais complexo. A oferta de cursos multidisciplinares torna-se mais crível em regimes anuais, na qual a participação do aluno torna a vivência do projeto mais correlacionável à prática profissional (GRIGG, 2018).

\section{CONCLUSÃO}

Dos 9 fatores institucionais modelados, 5 influenciam diretamente o desempenho de estudantes de engenharia civil no ENADE (Didática, Qualificação, Tempo, Organização e Jurisdição). Destes, a jurisdição administrativa dos cursos tem o maior peso de influência, com cursos federais e estaduais tendo melhores desempenhos que cursos municipais, seguida pela qualificação docente, organização administrativa (com instituições menores se sobrepondo às universidades), didática docente e tempo de funcionamento. Apesar dos fatores Dedicação, Vagas, Currículo e Regime não demonstrarem influência direta sobre o desempenho estudantil no ENADE, não se descarta a possibilidade destes auxiliarem o desempenho de alunos durante a graduação.

A limitação deste estudo consiste no universo amostral escolhido (cursos de graduação em engenharia civil ofertados por instituições públicas que tenham participado do ENADE 
2017) e no escopo orientado de investigação (Fatores institucionais ligados à docência e instituição ofertante). Estudos futuros e complementares podem discutir fatores socioeconômicos, demográficos e geográficos que impactam o desempenho desta amostra, ou ainda, expandi-la para instituições privadas de ensino.

Depreende-se que o objetivo desta pesquisa foi alcançado na medida que foram identificados os fatores institucionais que de fato influenciam no desempenho estudantil no ENADE por parte dos cursos públicos de engenharia civil. Com isso, espera-se contribuir com a teoria de estudos quantitativos de desempenho acadêmico no ENADE por estudantes de engenharia, em especial a modalidade civil, introduzindo novos protocolos estatísticos que incorporam maior robustez e credibilidade científica às predições. Existem também contribuições práticas, relacionadas ao auxílio de planejadores educacionais no desenvolvimento de protocolos de análise e avaliação de cursos de ensino superior no Brasil, em especial quanto aos seus desempenhos no ENADE, de modo a otimizar o sistema de monitoramento e acreditação atual.

\section{Agradecimentos}

O primeiro autor agradece à Coordenação de Aperfeiçoamento de Pessoal de Nível Superior (CAPES) pelo suporte financeiro concedido para realização desta pesquisa.

\section{REFERÊNCIAS}

CLEFF, Thomas. Applied Statistics and Multivariate Data Analysis for Business and Economics: A modern approach using SPSS, Stata, and Excel. $1^{\text {a }}$ edição, New York: Springer. 2019.

CORDEIRO, J. S. et al. Um futuro para a educação em engenharia no Brasil: desafios e oportunidades. Revista de Ensino de Engenharia, v. 27, n. 3, p. 69-82, 2008.

FORCAEL, E. et al. Application of Problem-Based Learning to Teaching the Critical Path Method. Journal of Education in Civil Engineering, v. 141, n. 3, 04014016, 2015. DOI: http://dx.doi.org/10.1061/(ASCE)EI.1943-5541.0000236.

GRIGG, N. S. Civil Engineering Workforce and Education: Twenty Years of Change. Journal of Education in Civil Engineering, v. 144, n. 4, 04018010, 2018. DOI: https://doi.org/10.1061/(ASCE)EI.1943-5541.0000387.

HAIR, Joseph et al. Multivariate Data Analysis. 8ª edição. Hampshire: Cengage. 2018.

INEP - Instituto Nacional de Estudos e Pesquisas Educacionais Anísio Teixeira. Banco de dados do ENADE 2017. Disponível em: http://portal.inep.gov.br/web/guest/microdados. Acesso em 05 fev. 2020.

LEITÃO, Marcílio de Alencar Sá. A transição de paradigmas no ensino de engenharia. In: XXIX Congresso Brasileiro de Ensino de Engenharia, 2001, Porto Alegre. Anais. Porto Alegre, 2013. p. 378-382.

MEC - Ministério da Educação. Banco de dados dos cursos públicos de engenharia civil. Disponível em: http://emec.mec.gov.br/. Acesso em 10 abr. 2020. 
MOREIRA, Ana Maria. Fatores institucionais e desempenho acadêmico no ENADE: um estudo sobre os cursos de Biologia, Engenharia Civil, História e Pedagogia. 2010. Tese (Doutorado) - Faculdade de Educação, Universidade de Brasília, Brasília, 2010.

PEREIRA, Sheila Regina dos Santos. Determinantes da equidade no ensino superior: uma análise da variabilidade dos resultados do Enade no desempenho de cotistas e não cotistas. Tese (Doutorado) - Faculdade de Educação, Universidade Federal da Bahia, Salvador, 2017.

RIVERA-RODRIGUEZ, J. C. Preliminary review of two curricular Civil Engineering programs in Bogota - Colombia. Revista Educación en Ingeniería, v. 15, n. 29, p. 83-88, 2017. DOI: http://dx.doi.org/10.26507/rei.v15n29.1077.

RUF - Ranking Universitário Folha. Programas públicos de engenharia civil. Banco de dados. Disponível em: http://ruf.folha.uol.com.br/2017/. Acesso em 18 fev. 2020.

SANKARAN, M.; MOHANTY, S. Student perception on achieved graduate attributes and learning experiences: a study on undergraduate engineering students of India. Int. J. Cont. Engineering Education and Life-Long Learning, v. 28, n. 1, p. 77-98, 2018. DOI: https://dx.doi.org/10.1504/IJCEELL.2018.090247.

SILVA, Flávia Guimarães Menezes. Desempenho dos estudantes no Enade: influenciadores ambientais e organizacionais sob a luz da Sociologia da Educação de Bourdieu. Dissertação (Mestrado) - Faculdade de Administração, Universidade Salvador, Salvador, 2017.

\title{
WHAT INFLUENCE THE STUDENT PERFORMANCE IN ENADE? QUANTITATIVE INVESTIGATION IN PUBLIC UNDERGRADUATE PROGRAMS IN CIVIL ENGINEERING IN BRAZIL
}

\begin{abstract}
This study aimed to identify which institutional factors influence the civil engineering student performance from public institutions in the ENADE. Inferential methods involving multiple linear regression were conducted to modelling the 9 variables interaction, associated to licensure and institution in 199 undergraduate programs across Brazil. After statistical validation, 5 associated variables to professor qualification, professor didactics, time of program functioning, academic organization and administrative jurisdiction performed valid correlation to the ENADE performance. Non valid variables, like professor's time dedication, number of admitted students, curricular workload and courses offering regime could not directly influence the ENADE performance, however, it does not discard their possible influences in the undergraduate performance at all. The theoretical adds concern the quantitative approach of engineering student performance in the ENADE, as well as the practical contributions to the development of educational politics to evaluate undergraduate programs in engineering across Brazil.
\end{abstract}

Keywords: Education in civil engineering. linear regression. ENADE. 